\title{
Etiologies of Nodular Skin Lesions in Passerines: A Case Series
}

\author{
Jeffery N. Carey ${ }^{1,2}$, Julia Lankton, DVM, DACVP $3^{*}$, and Erica A. Miller, DVM ${ }^{1}$ \\ ${ }^{1}$ University of Pennsylvania School of Veterinary Medicine, Philadelphia, Pennsylvania \\ ${ }^{2}$ University of Pennsylvania Graduate Group in Biochemistry and Molecular Biophysics, Philadelphia, \\ PENNSYLVANIA \\ 3 University of Tennessee College of Veterinary Medicine, Knoxville, Tennessee \\ *Current address: USGS-National Wildlife Health Center, Madison, Wisdconsin
}

\begin{abstract}
Nodular skin lesions often are observed in passerines presenting to wildlife rehabilitators. Although these lesions may appear grossly similar, their etiologies can be highly diverse, which confounds appropriate diagnosis and treatment. Presented here is a case series describing similar nodular skin lesions caused by avipoxvirus, aspergillosis, and follicular cysts, with the purpose of alerting rehabilitators to the variety of disease processes that can lead to grossly similar lesions and to the diagnostics available for their differentiation. Accurate diagnosis is expected to lead to more appropriate and targeted therapeutic protocols and improved prognoses.
\end{abstract}

Keywords: Skin lesions, nodular lesions, poxvirus, aspergillosis, follicular cysts, passerines

\section{INTRODUCTION}

Unfortunately, for rehabilitators and veterinarians, nodular skin lesions can appear grossly very similar, even if the underlying disease processes are dissimilar, and definitive diagnosis can be made only after time- and resource-consuming diagnostics such as histopathology or culture. Consequently, empirical treatment often is initiated before a definitive diagnosis is reached. For empirical treatment to be successful, the differential diagnosis list must be complete and accurate. To aid in the construction of a thorough

Jeffery N. Carey is sixth-year VMD-PhD student at the University of Pennsylvania. He has volunteered with Tri-State Bird Rescue \& Research, Inc., in Newark, DE and Flint Creek Wildlife Rehabilitation in Chicago, IL.

Julia Lankton, DVM, DACVP completed her residency training in pathology at the University of Tennessee, was a clinical instructor at the University of Florida and Disney's Animal Kingdom, and currently works as a pathologist at the US Geological Survey's National Wildlife Health Center in Madison, WI.

Erica A. Miller, DVM is chief veterinarian for Brandywine Zoo in Wilmington, DE, is an Adjunct Associate Professor at the University of Pennsylvania School of Veterinary Medicine, and works for the New Jersey Division of Fish \& Wildlife. She also is a past NWRA board member and served as Secretary. differential diagnosis list for passerines presenting with nodular skin lesions, cases illustrating three diseases that can manifest with similar clinical cutaneous signs are described here. All cases were presented to Tri-State Bird Rescue and Research (TSBRR) in Newark, DE in 2009.

\section{Case 1: Avipoxvirus Infection in a Brown} Thrasher (Toxostoma rufum). The Poxviridae family consists of large, enveloped DNA viruses that have evolved to infect a wide range of vertebrate and some arthropod species (Bolte, Meurer, and Kaleta 1999; Weli et al 2004). The avipoxviruses comprise a genus within this family that is globally distributed and known to infect over 232 species of wild birds (Bolte et al 1999). Birds can contract the virus by direct contact, aerosol, insect bites, contaminated food and water, or exposure to viral particles persisting in the environment (Ha et al 2013; Joshi et al 2012). The host specificity for individual species of avipoxvirus is variable (Thiel et al 2005).

Avipoxvirus infection can cause multiple forms of disease (Bolte et al 1999; Joshi et al 2012), but only the cutaneous form is discussed here. Cutaneous lesions are characterized by proliferative dermal nodules primarily distributed over unfeathered skin that can ulcerate and harden into thick crusts (Bolte et al 1999; Ha et al 2013; Joshi et al 2012; Parker et al 2011; Thiel et al 2005). Diagnosis is made by polymerase chain reaction (PCR) for viral genomic sequences (Ha et al 2013; Huw Lee and Hwa Lee 1997; Parker et al 2011; Thiel et al 2005), the visualization of eosinophilic intracellular inclusion bodies (Bollinger bodies) on histopathology (Joshi et al 2012; Parker et al 2011), electron microscopy (Bolte et al 1999; Weli et al 2004), virus isolation (Bolte et al 1999), or serology (Huw Lee and Hwa Lee 1997). 
There is no treatment for avipoxvirus infection outside of supportive care, but vaccination is effective for prevention of some strains (fowlpox, pigeon pox, and canarypox) (Bolte et al 1999; Marruchella and Todisco 2010). Mortality resulting from the disease is variable and is dependent somewhat on virus and host species (Bolte et al 1999; Marruchella and Todisco 2010).

Case Report-A nestling female brown thrasher found on 29 June 2009 and fed baby bird diet by its finder was presented to TSBRR on 1 July. At admission, the bird's eyes and nares were clogged with diet, its bones were palpably soft, and it had one small, white, abscess-like lesion at the keel and one over the ventral abdomen. On 4 July, multiple nodules were noted at the cranial keel, and the bird was euthanized on 5 July.

On necropsy the bird was in good body condition. It had a small amount of abdominal fat and enlarged adrenal glands. Multiple, discrete, caseous nodules were present in the subcutis over the entire ventrum, especially over the right breast and left femoral region (Figure 1). The upper gastrointestinal tract was empty and mucoid fluid was present in the small intestine. No other gross lesions were observed. Two sections demonstrating skin lesions were preserved in ten percent formalin for histopathology.

Histopathology of the skin sections (Figure 2) revealed moderate to severe epidermal hyperplasia with parakeratotic hyperkeratosis, ballooning degeneration of keratinocytes, eosinophilic intracytoplasmic inclusions, and multifocal superficial bacterial aggregates. These lesions are consistent with avipoxvirus infection.

The gross appearance of the skin lesions in this case was not immediately suggestive of avipoxvirus infection, as the nodules were present on feathered skin, caseous, and lacked any regions of ulceration or crusting. However, histopathology revealed the classic signs of avipoxvirus and provided the diagnosis. Avipoxvirus should remain on the differential diagnosis list for nodular dermal lesions of any gross appearance until further diagnostics rule it out.

Figure 2. Photomicrograph of avipoxvirus lesion demonstrating acanthosis, parakeratotic hyperkeratosis, and ballooning degeneration of keratinocytes.

igure 1. Brown thrasher with caseous avipoxvirus lesions.

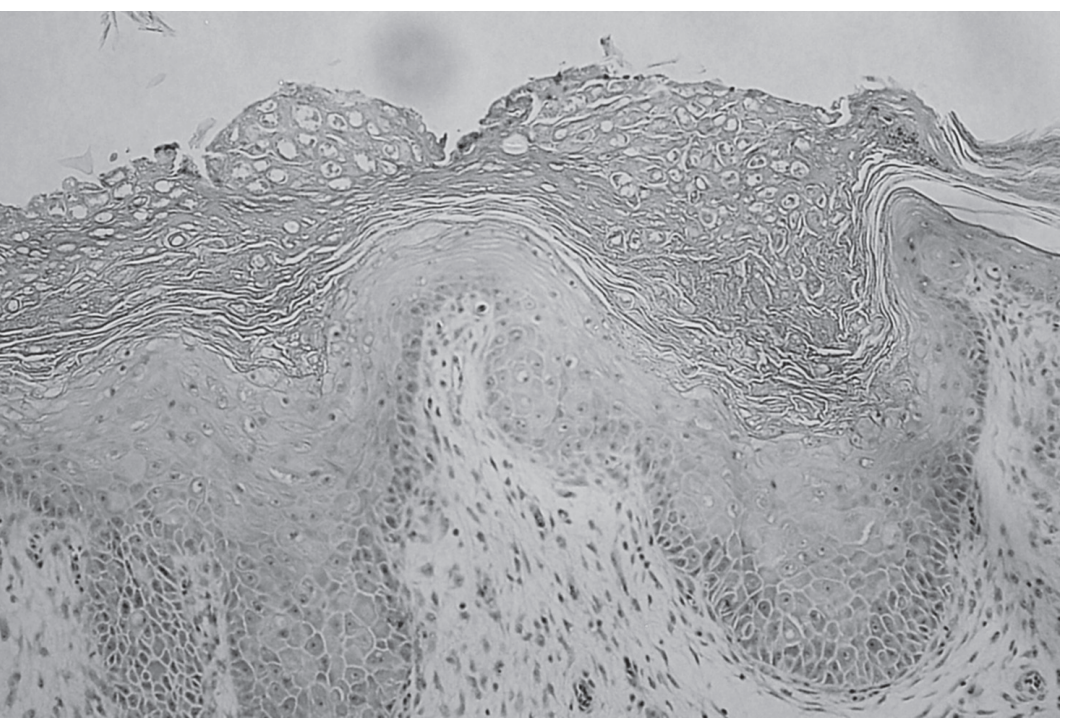

Case 2: Aspergillosis in a Carolina Chickadee (Poecile carolinensis). The most common fungal disease of birds is aspergillosis (França, Cray, and Shivaprasad 2012) and it is caused by molds in the genus Aspergillus, most frequently A. fumigatus (Jones and Orosz 2000). Aspergillus spp. are widely distributed and spores are ubiquitous in the environment (Low et al 2005). Infection occurs through inhalation of the spores, and A. fumigatus is thought to be the most commonly pathogenic species because its spores are too small to be captured and expelled effectively by the upper respiratory system (Beernaert et al 2010).

Aspergillus spp. most frequently initially colonize the host's lower respiratory system, causing nonspecific signs of illness (lethargy, anorexia) and/or respiratory compromise (dyspnea) (Tell 2005). Infection can 


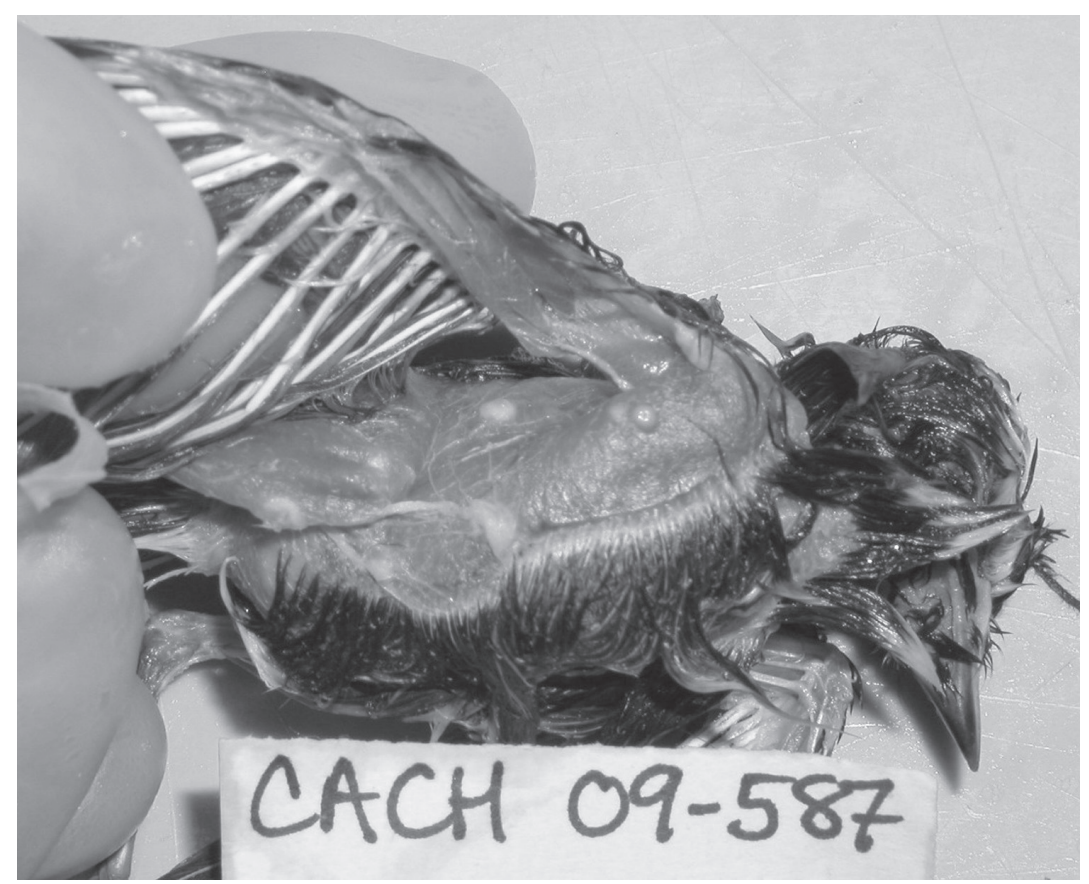

Figure 3. Carolina chickadee with dermal lesions resulting from disseminated aspergillosis.

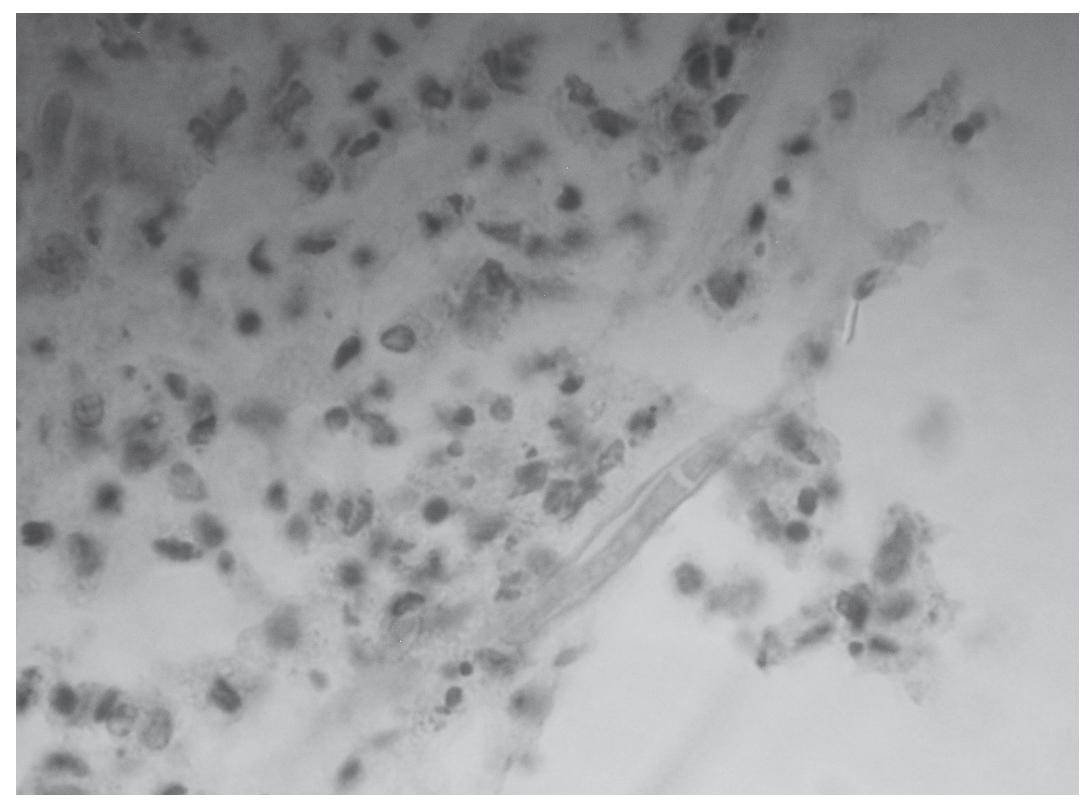

Figure 4. Photomicrograph of aspergillosis skin lesion illustrating a fungal hypha.
(Abrams et al 2001), but reports of mycotic dermatitis in birds are uncommon (Abrams et al 2001). Microscopic findings often include the presence of a mixed inflammatory cell population and fungal hyphae with septations and branching (Cray et al 2009). Granulomatous inflammation may be present (Jones and Orosz 2000; Low et al 2005). Radiographs and visualization via endoscopy are useful in providing supportive evidence for a diagnosis of gross lesions in the respiratory system, but no single test can diagnose aspergillosis definitively in every case (Beernaert et al 2010). Other available diagnostics include serology, hematology and blood chemistry, histopathology, cytology, fungal culture, and PCR (Beernaert et al 2010; Cray et al 2009; França et al 2012).

Aspergillosis has been treated successfully using antifungal chemotherapeutics systemically and/or topically, but the disease can be refractory to treatment because often it is advanced at the time of patient presentation, and it can be difficult for drugs to penetrate fungal granulomas (Beernaert et al 2010; Joseph 2000). Amphotericin B, azole antifungals, and 5-fluorocysteine all have been used to treat aspergillosis in birds; a useful summary of dosages and routes of administration in various avian species can be found in Beernaert et al (2010). Mortality related to aspergillosis in birds is high (Tell 2005).

Case Report-A male fledgling Carolina chickadee presented, along with two live nestmates, to TSBRR on 15 May 2009 after their parents were killed by a house sparrow. The patient

be acute or chronic, depending on the bird's immunocompetence and the dose of infectious spores (Jones and Orosz 2000), and can remain localized or progress to a disseminated form (Tell 2005). Although aspergillosis is considered primarily a respiratory disease, any body system can be affected by the disseminated fungus, including eyes, central nervous system, skin, skeleton, and coelomic organs (Beernaert et al 2010; França et al 2012; Jones and Orosz 2000).

Gross cutaneous lesions of aspergillosis that have been described include epidermal cysts (Suedmeyer, Bermudez, and Fales 2002), thickening, and crusts was bright, alert, and responsive, and seemed to thrive for approximately three weeks. On 7 June, it appeared weak and dehydrated. Examination revealed multiple yellow, caseous, 1 to $4 \mathrm{~mm}$ dermal nodules over the legs, sides of body, and axillae (Figure 3). The bird was euthanized the same day. The bird's nestmates remained healthy and were released.

In addition to the skin lesions, necropsy revealed caseous, fibrinous masses filling the lungs and a possible nodule in the brain. Four whole millet seeds were found in the ventriculus. No other abnormalities were observed. Skin, brain, and lung samples were collected 
and fixed in ten percent formalin for histopathology.

Microscopy of the lung tissue showed multifocal, marked necrotizing heterophilic pneumonia with intralesional fungal hyphae. Microscopy of the skin lesions showed widespread, multifocal, marked ulcerative and necrotizing heterophilic dermatitis, also with intralesional fungal hyphae (Figure 4). The hyphae in both lung and skin were septate with parallel walls and dichotomous branching. No significant microscopic findings were detected in the brain tissue. The lesions are consistent with disseminated aspergillosis.

While skin lesions are not classically associated with aspergillosis, in its disseminated form this disease can attack any part of the body. Consequently, aspergillosis is an appropriate differential diagnosis for nodular skin lesions, and empirical antifungal therapy would be appropriate if the overall clinical picture supports a diagnosis of aspergillosis, or if other attempted empirical therapies have failed. Of note is that cutaneous aspergillosis has been successfully diagnosed in humans through fine needle aspiration of skin nodules (Venugopal and Venugopal 2012). If this technique could be adapted to avian patients with suggestive nodular lesions, it could be used to support the clinical suspicion of disseminated aspergillosis before initiation of antifungal therapy.

\section{Case 3: Follicular Cysts in a Northern Cardinal (Cardinalis} cardinalis). Follicular cysts occur when the feather follicle becomes occluded and the developing feather is unable to emerge (Harrison 2003). There are many possible causes of cyst formation, including trauma, infection, genetic predisposition, and neoplasia (Couvillion, Maslin, and Montgomery 1990; Hafner et al 1991; Harrison 2003). Etiologic diagnosis can be a challenge and is tentatively achieved through exclusion of infectious causes along with histopathological examination of lesions (Couvillion et al 1990; Mutinelli et al 2008).

Follicular cysts grossly appear as hard nodules of variable size that may be irregularly shaped and contain a dry or caseous, tan or yellow material

(Couvillion et al 1990; Mutinelli et al 2008). The microscopic appearance can vary based on the etiology of the lesion, but regardless of cause the cyst has a core of keratin, necrotic keratinocytes, and sebaceous secretions (Couvillion et al 1990; Hafner et al 1991).

Optimally, treatment is directed against the underlying cause as well as the cyst itself. Options for managing a cyst include complete surgical excision, lancing, fulguration, and ablation, but only surgical excision has been demonstrated to remove a cyst without recurrence (Hafner et al 1991). Follicular cysts can be irritating to the bird (Harrison 2003; Mutinelli 
et al 2008), but do not inherently impose any physical or behavior restrictions on the animal (Mutinelli et al 2008) and tend to be self-limiting, even if neoplastic in origin (Hafner et al 1991). The keratinaceous material in the cyst provides an attractive substrate for secondary bacterial colonization (Hafner et al 1991; Mutinelli et al 2008), but it is unknown whether this predisposes the bird to bacterial dermatitis or if there are other negative impacts on survival fitness associated with the cystic disruption of normal integumentary structure.

Case Report-An adult female northern cardinal was brought to TSBRR after being found by a cat on 12 June 2009. The bird was missing most body feathers, though the tail was intact, and the skin was dry and wrinkly. The skin over the ventral neck contained dark nodules (Figure 5). The bird presented with multiple wounds on the chest, back, and sides, and was euthanized on arrival.

On necropsy, the spleen was enlarged $(15.4 \times$ $18.5 \mathrm{~mm}$ ) and the lateral half of the left lung was dark red and consolidated (demonstrated by its sinking in water). The ventriculus contained normal ingesta. There was some red speckling in the calvarium, but the brain appeared grossly normal. There were no other gross lesions, and multiple skin scrapings revealed no etiologic agents (neither parasitic nor microbial) for the feather loss or skin nodules. Skin biopsies were collected and fixed in ten percent formalin.

Histopathology showed that feather follicles of the ventral neck were multifocally dilated and contained concentric rings of lamellar keratin (Figure 6), indicating follicular cysts. Other follicles from the biopsied areas were frequently misshapen and irregular and contained abundant keratin, but the specimens were not diagnostic for primary follicular dysplasia versus a secondary follicular disorder. There also was moderate, diffuse hyperkeratosis and multifocal areas of dermal hemorrhage, with the hemorrhage likely associated with the history of trauma.

The nodular lesions described in this case most likely were caused by a defect in the normal functioning of feather follicles rather than by an infectious agent. Given the widespread feather loss and severe cat-inflicted wounds, euthanasia was warranted in this case. Uncomplicated follicular cysts, however, are reasonably treated by physical removal, though they may recur (Hafner et al 1991). In general, follicular cysts should remain on the differential diagnosis list for cutaneous nodules in birds that appear healthy overall, especially if the nodules are clearly located at or adjacent to feather follicles.

\section{SUMMARY}

Three cases illustrating three drastically different causes of similar gross lesions are described. By no means should these cases be considered comprehensive for the possible causes of nodular skin lesions in passerines; other common diseases that can cause nodular skin lesions in passerines include, for instance, follicular mites and bacterial folliculitis. The above cases should be approached instead as examples of how knowing the possible etiologies of a common lesion can lead to intelligent construction of a differential diagnosis list, which then can be used to guide diagnostic and treatment decisions. For an individual rehabilitator, these decisions differ for each case, perhaps being as comprehensive as molecular diagnostics and biopsy submission for birds with nodular skin lesions, or as simple as implementing increased biosecurity measures and providing enhanced monitoring to avipoxvirus suspects. In each of the above cases, the disease was too advanced or complicated to prevent euthanasia, but the hope of postmortem diagnosis is for increased vigilance and preparedness for the future.

\section{LITERATURE CITED}

Abrams, G. A., J. Paul-Murphy, J. C. Ramer, and C. J. Murphy. 2001. Aspergillus Blepharitis and Dermatitis in a Peregrine Falcon-Gyrfalcon Hybrid (Falco peregrinus $\times$ Falco rusticolus). Journal of Avian Medicine and Surgery. 15(2): 114-120.

Beernaert, L. A., F. Pasmans, L. Van Waeyenberghe, F. Haesebrouck, and A. Martel. 2010. Aspergillus Infections in Birds: A Review. Avian Pathology. 39(5): 325-331.

Bolte, A. L., J. Meurer, and E. F. Kaleta. 1999. Avian Host Spectrum of Avipoxviruses. Avian Pathology. 28(5): 415-432.

Couvillion, C. E., W. A. Maslin, and R. M. Montgomery. 1990. Multiple Feather Follicle Cysts in a Wild Turkey. Journal of Wildlife Diseases. 26(1): $122-124$.

Cray, C., et al. 2009. Galactomannan Assay and Plasma Protein Electrophoresis Findings in Psittacine Birds with Aspergillosis. Journal of Avian Medicine and Surgery. 23(2): 125-135.

França, M., C. Cray, and H. L. Shivaprasad. 2012. Serologic Testing for Aspergillosis in Commercial Broiler Chickens and Turkeys. Avian Diseases. 56(1): 160-164.

Ha, H., M. Alley, L. Howe, I. Castro, and B. Gartrell. 2013. Avipoxvirus Infections in Brown Kiwi (Apteryx mantelli). New Zealand Veterinary Journal. a61(1): 49-52. 
Hafner, S., B. G. Harmon, G. N. Rowland, and R. G. Stewart. 1991. Spontaneous Regression of "Dermal Squamous Cell Carcinoma" in Young Chickens. Avian Diseases. 35(2): 321-327.

Harrison, G. J. 2003. Microsurgical Procedure for Feather Cyst Removal in a Citron-crested Cockatoo (Cacatua sulphurea citrinocristata). Journal of Avian Medicine and Surgery. 17(2): 86-90.

Huw Lee, L., and K. Hwa Lee. 1997. Application of the Polymerase Chain Reaction for the Diagnosis of Fowl Poxvirus Infection. Journal of Virological Methods. 63(1-2): 113-119.

Jones, M. P., and S. E. Orosz. 2000. The Diagnosis of Aspergillosis in Birds. Seminars in Avian and Exotic Pet Medicine. 9(2): 52-58.

Joseph, V. 2000. Aspergillosis in Raptors. Seminars in Avian and Exotic Pet Medicine. 9(2): 66-74.

Joshi, S., M. Mudasir, D. Sharma, and R. Singh. 2012. Histopathological Study of Cutaneous Form of Avipoxvirus Infection in Jungle Crow (Corvus macrorhynchos). Veterinary World. 5(10): 628-630.

Low, M., A. Berggren, K. J. Morgan, and M. R. Alley. 2005. Aspergillosis in a North Island Robin (Petroica longipes). New Zealand Veterinary Journal. 53(6): 462-464.

Marruchella, G., and G. Todisco. 2010. Pathology in Practice. Poxvirus Infection. Journal of the American Veterinary Medical Association. 237(10): 1139-1141.

Mutinelli, F., M. Corro, S. Catania, and E. Melchiotti. 2008. Multiple Feather Follicle Cysts in a Moroseta Hen (Gallus gallus). Avian Diseases. 52(2): 345-347.
Parker, P. G., et al. 2011. 110 Years of Avipoxvirus in the Galapagos Islands. PLoS ONE. 6(1):e15989.

Suedmeyer, W. K., A. J. Bermudez, and W. H. Fales. 2002. Treatment of Epidermal Cysts Associated with Aspergillus Fumigatus and Alternaria Species in a Silky Bantam Chicken. Journal of Avian Medicine and Surgery. 16(2): 133-137.

Tell, L. A. 2005. Aspergillosis in Mammals and Birds: Impact on Veterinary Medicine. Medical Mycology. 43 Suppl 1: S71-S73.

Thiel, T., et al. 2005. Characterization of Canarypox-like Viruses Infecting Endemic Birds in the Galápagos Islands. Journal of Wildlife Diseases. 41(2): 342-353.

Venugopal, T. V., and P. V. Venugopal. 2012. Primary Cutaneous Aspergillosis from Tamilnadu Diagnosed by Fine Needle Aspiration Cytology. Medical Mycology Case Reports. 1(1): 103-106.

Weli, S. C., M. I. Okeke, M. Tryland, O. Nilssen, and T. Traavik. 2004. Characterization of Avipoxviruses from Wild Birds in Norway. Canadian Journal of Veterinary Research. 68(2): 140-145. (NRR

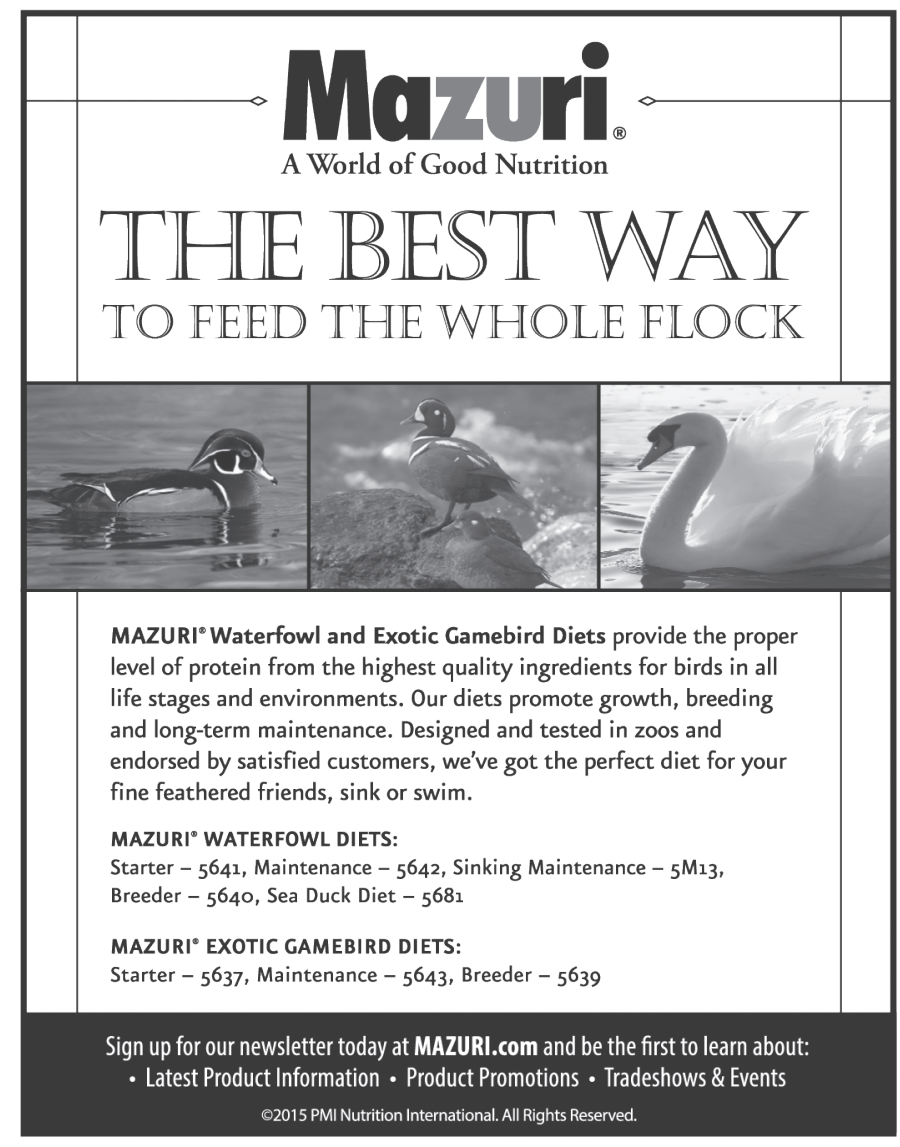

